\title{
Atomic Scale Characterization of Oxygen Vacancy Dynamics by In -Situ Reduction and Analytical Atomic Resolution STEM
}

\author{
R.F. Klie** N. D. Browning* and Y. Zhu ${ }^{1}$ \\ * University of Illinois at Chicago, 845 West Taylor Street, Chicago, IL 60607-7059 \\ ${ }^{1}$ Brookhaven National Laboratory, Dept. of Applied Science, Upton, New York 11973
}

In this study, we present nano-scale investigations of point defect dynamics in perovskite oxides by correlated atomic resolution high angle annular dark field imaging (HAADF) and electron energy loss spectroscopy (EELS). The point defect dynamics and interactions during in-situ reduction in the microscope column are analysed. In particular, oxygen vacancy creation, diffusion and clustering are studied, as oxygen vacancies comprise the majority of the point defects present in these perovskite oxide systems [1].

The results have been acquired using the JEOL2010F, a STEM/TEM, equipped with a $200 \mathrm{keV}$ field emission gun, a high angle annular dark field detector and a post column Gatan imaging filter (GIF). The combination of the $\mathrm{Z}$-contrast and EELS techniques [2] allows us to obtain direct images (spatial resolution of $2 \AA$ ) of the atomic structure and to correlate th is information with the atomically resolved EELS information ( $3 \mathrm{~s}$ acquisition time, $1.2 \mathrm{eV}$ energy resolution). In-situ heating of the material is performed in a Gatan double tilt holder with a temperature $\mathrm{r}$ ange of $300 \mathrm{~K}$ $-773 \mathrm{~K}$ at an oxygen partial pressure of $P_{\mathrm{O}_{2}}=5 * 10^{-8} \mathrm{~Pa}$. [3]

Figure 1 shows the oxygen ordering process in $(\mathrm{La}, \mathrm{Sr}) \mathrm{FeO}{ }_{3}$. After an initial reduction process, the anion non-stoichiometric Brownmillerite (BM) phases are formed, due to or dering of newly created oxygen vacancy. Upon in -situ reduction in the microscope column at $724 \mathrm{~K}$ for 12 hours, the BM micro-domains progressively grow in size, but their orientation and boundaries appear to be random in the bulk of the grain (Figure 1a). Further reduction at $1073 \mathrm{~K}$ for 3 hours leads to the formation of larger domains $(\sim 20 \mathrm{~nm})$ (figure $1 \mathrm{~b}$ ), caused by the selective reduction of the tetrahedrally coordinated Fe-O columns. Finally, the ex -situ reduction at $1073 \mathrm{~K}$ in $P_{\mathrm{O}_{2}}=1 * 10^{-10} \mathrm{~Pa}$ causes the material to decompose, by forming Aurivillius -like stacking faults (figure 1c), which is attributable to the collapse of the reduced $\mathrm{Fe}-\mathrm{O}$ columns and a structural rearrangement of perovskite layer sandwiched between two rocksalt Fe-O layers.

Figure 2 shows a $58^{\circ}$ [001] grain boundary in nominally undoped $\mathrm{SrTiO} \quad 3$ after $3 \mathrm{~h}$ of in - situ reduction at $724 \mathrm{~K}$. [4] The symmetric grain boundary exhibits structural units [5] with half columns of strontium forming the center of the dislocation cores. Figure $2 b$ dis plays the sum of 15 background-subtracted and multiple scattering deconvoluted spectra (normalized to $500 \mathrm{eV} \quad-530 \mathrm{eV}$ ) that show the differences between the bulk and the grain boundary oxygen concentration. The lower intensity, the smaller fine-structure of the boundary oxygen K-edge as well as the shift and decrease in the $\mathrm{L}_{3} / \mathrm{L}_{2}$ intensity ratio of the Ti edge suggest a significantly segregation of oxygen vacancy at the interface under such reducing conditions.

Current instrumentation upgrades at our labs will include the incorporation of an aberration corrector in the VG HB601 and developing atomic resolution Z -contrast imaging capabilities in the JEOL

- current address: Brookhaven National Laboratory, Dept. of Applied Science, Upton, New York 11973 
JEM3000. With these instrument improvements we will be capable of decreasing the electron probe size to $<1 \AA$ while increasing the current density by a factor of 10 and the energy resolution by a factor of 2. Future work will concentrate on column -by column EELS analysis of these ordered vacancy phases in the bulk and the oxygen vacancy and dopant segregation at $g$ rain boundaries of perovskite oxides at both room-temperature and highly reducing conductions. [6]

References:

1. S. Ling, Phys. Rev. B, 49 (1994)

2. E. M. James and N. D. Browning, Ultramicroscopy 78 (1999)

3. R.F. Klie and N. D. Browning, J. Electron Microsc. (in print)

4. R.F. Klie, N. D. Browning, Appl. Phys. Lett., 77, 23, 3737 (2000)

5. N. D. Browning and S.J. Pennycook, J. Phys D 29, 1779 (1996).

6. This research was sponsored by the U.S. Department of Energy under grant numbers DE-FG0296ER45610, No. DE-FC26-99FT40054 and No. DE-AC02-98CH10886. The JEOL 2010F microscope was purchased with support from the National Science Foundation (NSF-DMR9601792), and is operated by the Research Resources Center at the University of Illinois at Chicago.

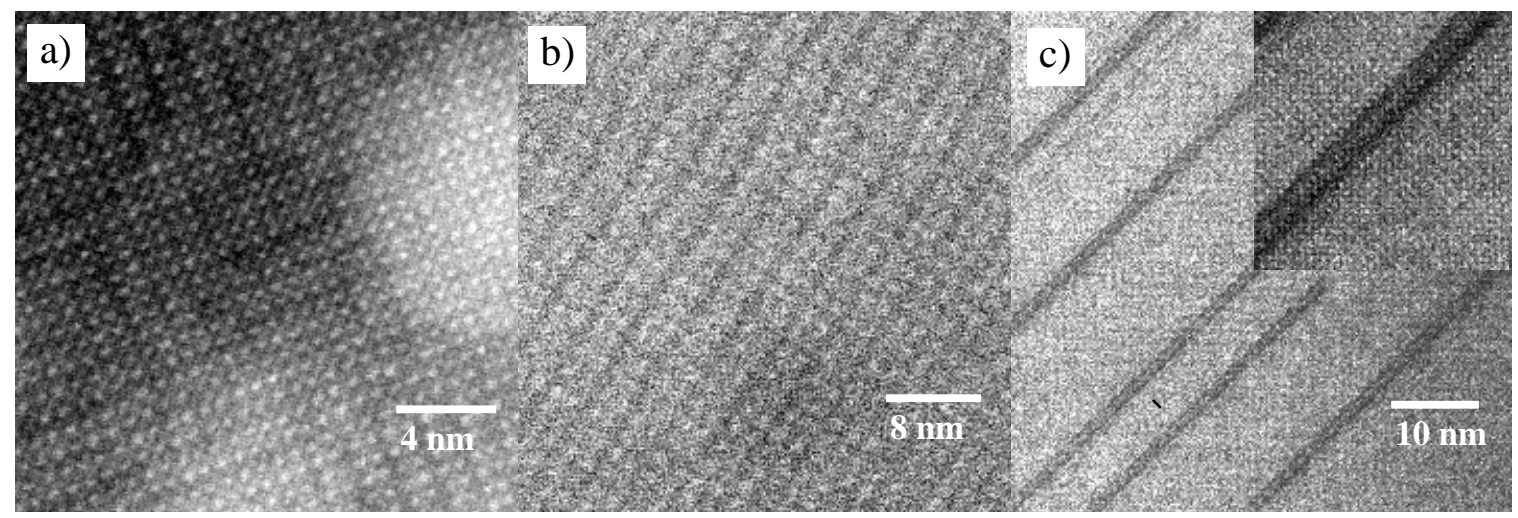

Figure 1: a) Z-Contrast image of ( $\mathrm{La}, \mathrm{Sr}) \mathrm{FeO}_{3}$ at $724 \mathrm{~K}$ with clearly visible ordered domain. b) ZContrast image of ( $\mathrm{La}, \mathrm{Sr}) \mathrm{FeO}_{3}$ at $724 \mathrm{~K}$ after heating to $1024 \mathrm{~K}$ for 3 hours. c) Z-Contrast image of $(\mathrm{La}, \mathrm{Sr}) \mathrm{FeO}_{3}$, previously treated at $800^{\circ} \mathrm{C}$ in $10^{-10} \mathrm{~Pa}$ showing stacking faults and ordered structures
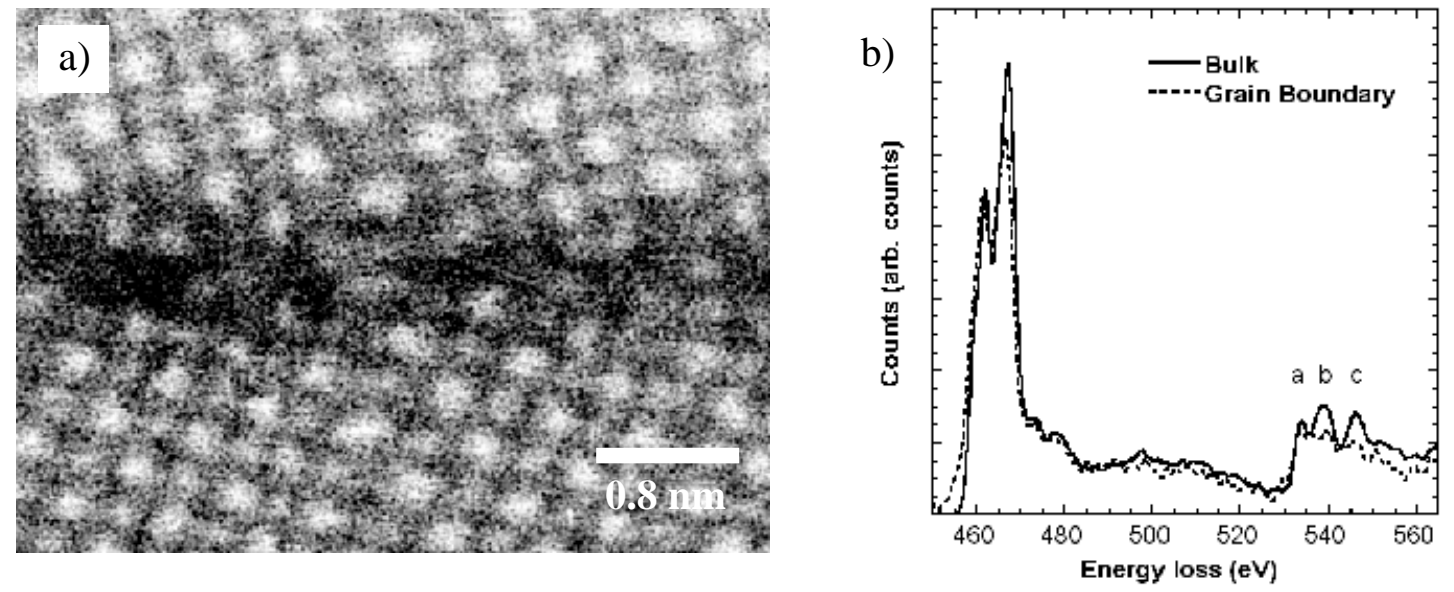

Figure 2: a) Z-Contrast image of a $\mathrm{SrTiO}_{3} 58^{\circ}$ [001] grain boundary at $724 \mathrm{~K}$. b) EEL spectrum from the grain boundary and the bulk

email: rkflie@uic.edu 